\title{
Detección de la mutación S315T en el gen katg como estrategia para identificación de Mycobacterium tuberculosis resistente a isoniacida en un laboratorio de referencia
}

\author{
Zolay Romay, Nailet Arráiz, Alisbet Fuenmayor, Carmen Ramírez, Luis Rojas y Rafael Paris
}

\section{Detection of S315T mutation in the katg gene as a strategy for identification of isoniazid-resistant Mycobacterium tuberculosis in a reference laboratory}

Introduction: The emergence of strains of Mycobacterium tuberculosis resistant to drugs is a public health problem. Aim: To characterize the resistance to isoniazid (INH) in M. tuberculosis. Methods: Phenotypic and genotypic methods were used to determine the contribution of mutations at 315 codon of kat $G$ gene to the phenotypic expression of resistance. The analysis of susceptibility to antibiotics was performed by the proportional method of Canetti and nitrate reductase method.Genotypic analysis of INH resistance was performed by PCRRFLP. Results: 193 strains of $M$. tuberculosis from patients with respiratory symptoms were analyzed. Nineteen $(9.8 \%)$ strains resistant to INH were identified, of which $12(63.2 \%)$ showed resistance to other drugs. Genotypic analysis allowed to detect the mutation S315T in the kat G gene in 15 of 17 strains phenotypically resistant to INH, showing a sensitivity of $88.24 \%, 100 \%$ specificity, $100 \%$ positive predictive value, $92 \%$ negative predictive value and high concordance with phenotypic methods (kappa $=0.85(\mathrm{p}<0.01)$. Conclusion: The S315T mutation in the $k a t G$ gene is the predominant mechanism of INH resistance in our circulating strains. This feature could be used as a rapid diagnostic tool with potential to detect at least $88 \%$ of isoniazid resistant strains, with great impact on the therapeutic management of patients.

Key words: Mycobacterium tuberculosis, resistance to isoniazid, MDR-TB, nitrate reductase, katGS315T.

Palabras clave: Mycobacterium tuberculosis, resistencia a isoniacida, TB-MDR, nitratoreductasa, katGS315T.

\section{Introducción}

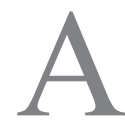
unque la tuberculosis (TBC) se consideraba una enfermedad de muy mal pronóstico por la inexistencia de estrategias de tratamiento efectivas, desde hace más de 50 años se introdujo la quimioterapia antituberculosa, con fármacos altamente eficaces contra el bacilo, como isoniacida (HIN) y rifampicina (RFP). En 1993, la Organización Mundial de la Salud (OMS) declaró la TBC como una emergencia sanitaria, y a partir de 1994 el Programa Mundial de Tuberculosis y la Unión Internacional contra TBC y Enfermedades Respiratorias, iniciaron un proyecto mundial para la vigilancia de la resistencia a los fármacos anti-tuberculosos. Sin embargo, la TBC se ubica actualmente dentro de las enfermedades infecciosas asociadas a un elevado índice de morbilidad y mortalidad en todo el mundo ${ }^{1-3}$.

En América Latina se producen entre $250.000 \mathrm{y}$ 300.000 nuevos casos y entre 20.000 y 25.000 muertes, anualmente ${ }^{3}$. Particularmente en Venezuela, para el año 2008 fueron notificados 6.173 casos nuevos de TBC, tanto pulmonares como extra-pulmonares, con una tasa de incidencia de 22,1 casos por 100.000 habitantes, por lo que el país se ubica dentro del grupo de países con moderada prevalencia en la región de las Américas ${ }^{4}$. Las entidades con tasas más altas de incidencia de TBC son: Delta Amacuro (69,14/100.000 habitantes), Distrito Capital (68,94/100.000 habitantes), Amazonas (42,05/100.000 habitantes), Portuguesa $(28,75 / 100.000$ habitantes), Sucre $(28,30 / 100.000$ habitantes $)$, Vargas $(27,82 / 100.000 \text { habitantes })^{4}$. En el Estado Zulia, durante el año 2009 se reportaron 730 casos nuevos de TBC (en todas sus formas), correspondiéndole así una tasa de 18,17 casos/100.000 habitantes 5 .

Esta problemática se complica por la circulación de cepas de M. tuberculosis multidrogoresistentes (TBC-MDR), que expresan resistencia a los fármacos considerados de primera línea para el tratamiento de la TBC, como lo son HIN y RFP ${ }^{6-9}$, así como también a la reciente aparición de cepas extremadamente resistentes (TBC-XDR), con resistencia añadida a fluoroquinolonas y a alguno de los tres fármacos considerados de segunda línea (amikacina, kanamicina y capreomicina) ${ }^{10}$.

A través de diversos estudios, se ha encontrado que las cepas resistentes a HIN y RFP ya han desarrollado resistencia a otros antimicrobianos, por lo cual la resistencia a estos antimicrobianos se considera un marcador para la detección de cepas de TBC-MDR ${ }^{11-13}$. La importancia del
Laboratorio Regional de Referencia de Tuberculosis. Maracaibo. Estado Zulia, Venezuela y Programa Regional de Control de Tuberculosis (ZR, RP).

Facultad de Medicina, Universidad del Zulia, Venezuela Centro de Investigaciones Endocrino-Metabólicas “Dr. Félix Gómez"

Sección de Biología Molecular (NA) Escuela de Bioanálisis (AF).

Laboratorio Nacional de Referencia de Tuberculosis y

Programa Nacional de Tuberculosis y Enfermedades Respiratorias Hospital el Algodonal. Caracas. Venezuela (CR, LR).

Conflicto de intereses: No existen conflictos de intereses para la ejecución del estudio y publicación de este manuscrito.

Este trabajo fue ejecutado por autogestión con recursos económicos de entes involucrados en el estudio: Coordinación del Programa Nacional de Tuberculosis y Enfermedades Respiratorias, Laboratorio Nacional de Referencia de Tuberculosis y Centro de Investigaciones EndocrinoMetabólicas Dr. "Félix Gómez", Facultad de Medicina, Universidad del Zulia.

Recibido: 28 de abril de 2012 Aceptado: 17 de septiembre de 2012

Correspondencia a: Nailet Arráiz Rodríguez. narraiz@1@yahoo.com; narraiz1@gmail.com 
estudio de estos marcadores reside en que la terapia de corta duración DOTS propuesta por la OMS deja de ser la mejor opción para el tratamiento de pacientes infectados con TBC-MDR y en la medida que la detección de una cepa multi-resistente se haga más temprano, mayor será la probabilidad de indicar un esquema terapéutico que incluya fármacos de segunda línea, contribuyendo a disminuir el riesgo de propagación de cepas de TBC-MDR y suministrar información epidemiológica valiosa para la ejecución y evaluación de los programas de control de TBC.

Ante la necesidad urgente de conocer a la mayor brevedad la susceptibilidad de las cepas de M. tuberculosis frente a los antimicrobianos de primera línea, la incorporación de ensayos genotípicos de resistencia a antimicrobianos se perfila como una opción que contribuiría al manejo terapéutico oportuno del paciente y a disminuir el riesgo de propagación de cepas de TBC-MDR.

En el caso particular de HIN, se han reportado mutaciones específicas en algunos genes, que explican la expresión fenotípica de resistencia a este fármaco. La isoniacida es una prodroga que, una vez captada por el bacilo, es activada por el sistema catalasa-peroxidasa y tiene acción bactericida al interferir con la biosíntesis de los ácidos micólicos de la pared celular de las micobacterias $^{11-13}$.

La ausencia de actividad catalasa, debida a mutaciones en el gen kat $G$, codificante de esta enzima, constituye uno de los principales mecanismos de resistencia a $\mathrm{HIN}^{12-15}$. Las mutaciones asociadas a resistencia a HIN se concentran en una región codificante del gen $\mathrm{katG}$, que comprende los codones 300 al $507^{13-15}$ y las cepas de M. tuberculosis portadoras de la mutación en el codón 315 del gen kat $G$ exhiben poca o ninguna actividad catalasa y son altamente resistentes a HIN (CIM > 32 $\mu \mathrm{g} / \mathrm{ml})^{16-21}$.

En el presente estudio se realizó el análisis fenotípico y genotípico de resistencia a HIN, en cepas de $M$. tuberculosis aisladas en los Laboratorios Regional y Nacional de Referencia de Tuberculosis, con la finalidad de evaluar la contribución de las mutaciones en el gen $\operatorname{kat} G$ a la expresión fenotípica de esa resistencia y la utilidad del análisis genotípico basado en la detección de la mutación katG-315 como una herramienta diagnóstica para determinar resistencia a HIN en aislados clínicos.

\section{Materiales y Métodos}

\section{Cepas bacterianas y análisis fenotípico}

Se analizaron 193 cepas de M. tuberculosis, provenientes de pacientes sintomáticos respiratorios atendidos en diversas regiones del país, las cuales fueron remitidas al Laboratorio Nacional de Referencia de Tuberculosis durante el período 2008-2011.
Todas las cepas fueron cultivadas en el medio de Lowenstein-Jensen, e identificadas como M. tuberculosis en base a su velocidad de crecimiento (lento, de 4 a 8 semanas), morfología colonial (colonias de color crema, no productoras de pimento, secas y rugosas) y un resultado positivo para la prueba de la niacina ${ }^{22}$. Para el control de calidad de todos los análisis de susceptibilidad se utilizó la cepa $\mathrm{H}_{37} \mathrm{R}_{\mathrm{v}}$ de $M$. tuberculosis, una cepa sensible a todos los agentes antituberculosos.

El análisis fenotípico de susceptibilidad a antimicrobianos se realizó a partir de subcultivos por el método de las proporciones de Canetti ${ }^{22}$ y el método indirecto de nitrato reductasa $a^{23,24}$. Para las pruebas de susceptibilidad se utilizaron concentraciones de $0,2 \mu \mathrm{g} / \mathrm{ml}$ y $40,0 \mu \mathrm{g} /$ $\mathrm{ml}$ de HIN y RFP, respectivamente. El protocolo de investigación fue aprobado por el Comité de Bioética del Centro de Investigaciones Endocrino-Metabólicas "Dr. Félix Gómez" de la Facultad de Medicina de la Universidad del Zulia.

\section{Prueba genotípica para la detección \\ de resistencia a isoniacida}

Para detectar mutaciones en el codón 315 del gen kat $G$ de $M$. tuberculosis, se utilizó la reacción de polimerasa en cadena (RPC), acompañada del análisis de polimorfismos de longitud de fragmentos de restricción (PLFR), técnica que fue seleccionada en este estudio y puede ser utilizada de rutina por su sencillez y menor costo que otras técnicas de detección de mutaciones, tales como secuenciación y análisis conformacional de ácidos nucleicos.

La extracción del ADN de las colonias micobacterianas se llevó a cabo utilizando un procedimiento de lisis enzimática previamente estandarizado ${ }^{25}$. Para la detección de la mutación en el codón 315 se utilizó la estrategia RPC-PLFR, utilizando los partidores katG2F (5'-GGTCGACATTCGCGAGACGTT-3) y katG2R (5'-CGGTGGATCAGCTTGTACCAG-3') para amplificar un fragmento de ADN de 377 pb contentivo del codón 315 correspondiente a una secuencia del gen $\mathrm{katG}$ de $M$. tuberculosis, de acuerdo al protocolo descrito previamente ${ }^{26}$.

Para las reacciones de amplificación, se preparó la mezcla de reacción en un volumen final de $50 \mathrm{ml}$, consistiendo en $5 \mathrm{ml}$ de tampón Taq ADN polimerasa 5X (Promega), 1,5 $\mu \mathrm{M}$ de $\mathrm{MgCl}_{2}, 25 \mathrm{mM}$ de cada partidor (katG2F- katG2R); $200 \mathrm{mM}$ de cada desoxiribonucleótido (dATP, dCTP, dGTP y dTTP) y 1 unidad de Taq ADN polimerasa (Promega). Se utilizó una desnaturalización inicial a $95^{\circ}$ por $10 \mathrm{~min}$; seguido de 25 ciclos de $30 \mathrm{seg}$ a $95^{\circ} \mathrm{C} ; 30 \mathrm{seg}$ a $68^{\circ} \mathrm{C}$ y $30 \mathrm{seg}$ a $72^{\circ} \mathrm{C}$. Se utilizó un termociclador MJ Research, modelo PTC-100. El producto amplificado (377 pb) se analizó por electroforesis en gel de agarosa al 1,5\% teñido con bromuro de etidio y visualizado en un transiluminador 
de luz UV. Como marcador de peso molecular se utilizó PCR Markers ${ }^{\mathrm{TM}}$ de Promega que incluye fragmentos de ADN de: 1.000, 750, 519, 310 y 155 pb.

Los productos de la RPC fueron sometidos a digestión con la enzima de restricción MspA11. La enzima de restricción reconoce la secuencia intacta del segmento de 377 pb amplificado y es capaz de cortar éste en dos fragmentos con una longitud de 309 pb y 68 pb, pero no es capaz de escindir los segmentos que presentan la mutación 315 (Figura 1). Los productos de digestión fueron analizados en geles de agarosa al $2 \%$, teñidos con bromuro de etidio.

\section{Análisis estadístico}

Se compararon los resultados obtenidos mediante el análisis fenotípico convencional de las proporciones y el método genotípico. Utilizando las fórmulas establecidas, se calculó la sensibilidad, especificidad y valores predictores positivo y negativo de la RPC-PLFR para detectar la mutación 315 del gen katG de $M$. tuberculosis. Se utilizó el estadístico kappa $(k)$ para estimar el grado de concordancia entre los resultados del análisis de resistencia a HIN por el método de las proporciones y los obtenidos mediante el análisis genotípico. Un valor $\mathrm{p}<0,05$ fue considerado significativo. La valoración del índice kappa, como estimador de la fuerza de la concordancia entre las variables se basó en la siguiente escala: < 0,20: pobre; 0,21-0,40: débil; 0,41-0,60: moderada; 0,61-0,80: buena; 0,81-1,00: muy buena.

\section{Resulltados}

De acuerdo al análisis fenotípico de resistencia por el método de las proporciones de 193 aislados clínicos, 19 de estas cepas $(9,8 \%)$ resultaron resistentes a HIN. Dos de las cepas resistentes a HIN no estuvieron disponibles para posteriores análisis, De las 17 cepas resistentes a HIN disponibles, 12 (70,6\%) exhibieron resistencia a alguno de los otros antimicrobianos probados, lo cual corresponde al 6,2\% del total de las cepas estudiadas, (Tabla 1). Se observó resistencia a RFP, otro fármaco de primera línea, en 11 de los 17 (64,7\%) aislados clínicos resistentes a HIN. Es importante resaltar que 6 aislados clínicos, es decir, 50\% de las cepas multi-resistentes y $3 \%$ del total de cepas resistentes evaluadas, presentaron resistencia a cuatro antimicrobianos.

Los resultados del análisis fenotípico de resistencia a HIN por el método indirecto de nitrato reductasa exhibieron una concordancia de $100 \%$ con los resultados obtenidos por el método de las proporciones.

El estudio genotípico de resistencia a HIN basado en la detección de la mutación S315T del gen katG, fue aplicado a 40 cepas: 23 sensibles a HIN y los 17 aislados clínicos que resultaron resistentes a este antimicrobiano por los métodos fenotípicos.
El análisis RPC-PLFR permitió detectar los segmentos de 309 pb y 68 pb, indicativo de la presencia del gen KatG no mutado, en las 23 cepas clasificadas como sensibles en los ensayos fenotípicos ( $100 \%$ de concordancia entre los métodos fenotípico y genotípico en cepas sensibles). Un segmento único de $377 \mathrm{pb}$, indicativo de la presencia de la mutación 315 en el gen $K a t G$, fue observado en 15 $(88,24 \%)$ de las 17 cepas clasificadas como resistentes por los estudios fenotípicos (Figura 2, Tabla 2). Sólo en

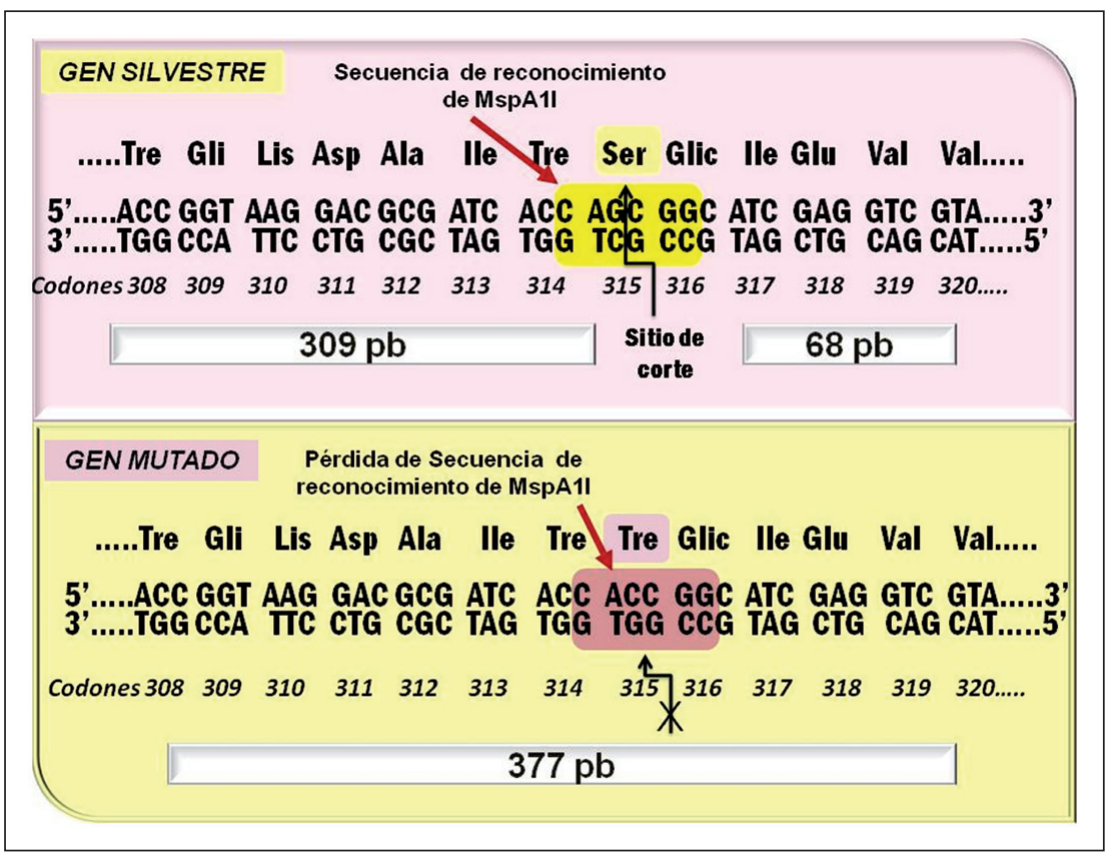

Figura 1. Secuencia de reconocimiento de la enzima y MspA1l y estrategia de detección de la mutación en el codón 315 del gen katG.

Tabla 1. Resultados del análisis de sensibilidad a fármacos anti-tuberculosos por el método de las proporciones

\begin{tabular}{|c|c|c|c|c|}
\hline \multirow[t]{2}{*}{ n de cepas } & \multicolumn{4}{|c|}{ Fármacos ensayados } \\
\hline & HIN & RFP & SM & ET \\
\hline 174 & s & S & S & $S$ \\
\hline 5 & R & S & $S$ & $\mathrm{~S}$ \\
\hline 4 & $\mathrm{R}$ & $\mathrm{R}$ & S & $S$ \\
\hline 1 & $\mathrm{R}$ & S & $R$ & $S$ \\
\hline 1 & $\mathrm{R}$ & $\mathrm{R}$ & S & $\mathrm{R}$ \\
\hline 6 & $R$ & $\mathrm{R}$ & $\mathrm{R}$ & $\mathrm{R}$ \\
\hline 2 & R & NE & $\mathrm{NE}$ & $\mathrm{NE}$ \\
\hline Cepa control & S & S & S & S \\
\hline
\end{tabular}


Figura 2. RPC-PLFR: PM: marcador de peso molecular; carriles $1,2,4,5,6,7,8,9$, $10,11,12,13$ y 14 : cepas sensibles (banda de $309 \mathrm{pb}$ ); carriles 15, 16 y 18: cepas resistentes (banda de $377 \mathrm{pb}$ ); carril 19: cepa control de $M$. tuberculosis $\mathrm{H}_{37} \mathrm{R}_{v}$.

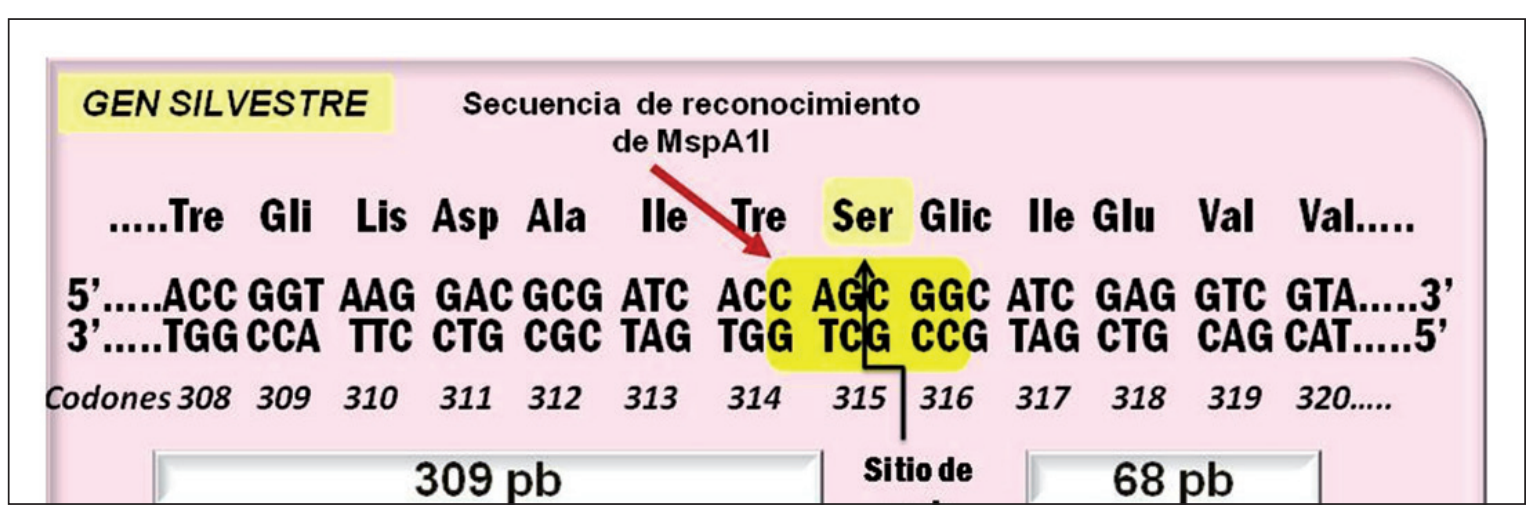

\section{Tabla 2. Caracterización fenotípica y genotípica de resistencia a isoniacida en cepas} de M. tuberculosis

\begin{tabular}{|c|c|c|c|}
\hline \multirow[t]{2}{*}{ n de cepas } & \multicolumn{2}{|c|}{ Métodos fenotípicos } & \multirow{2}{*}{$\begin{array}{c}\text { Método genotípico } \\
\text { RPC-PLFR }\end{array}$} \\
\hline & $\begin{array}{c}\text { M. de las } \\
\text { proporciones }\end{array}$ & $\begin{array}{l}\text { M. de nitrato } \\
\text { reductasa indirecto }\end{array}$ & \\
\hline 23 & Sensible & Sensible & No mutada \\
\hline 15 & Resistente & Resistente & Mutada \\
\hline 2 & Resistente & Resistente & No mutada \\
\hline
\end{tabular}

Tabla 3. Contribución de las mutaciones en el gen katG a la expresión de resistencia a isoniacida en $M$. tuberculosis en la literatura científica

\begin{tabular}{|c|c|c|c|}
\hline Autores & $\begin{array}{l}\text { Población, año de } \\
\text { publicación }\end{array}$ & $\begin{array}{l}\text { Número de cepas HIN-R } \\
\text { evaluadas }\end{array}$ & $\begin{array}{l}\text { Porcentaje } \\
\text { (\%) }\end{array}$ \\
\hline Caws Maxine y cols ${ }^{26}$ & Vietnam, 2007 & 47 & 80,0 \\
\hline Mathuria J y cols ${ }^{20}$ & India, 2009 & 121 & 72,7 \\
\hline Ahmad Sy cols ${ }^{36}$ & Kuwait, 2009 & 60 & 78,7 \\
\hline Yao C y cols ${ }^{29}$ & China, 2010 & 50 & 82,0 \\
\hline Zhang L y cols ${ }^{16}$ & China, 2010 & 26 & 84,6 \\
\hline Perizzolo y cols ${ }^{14}$ & Brasil, 2011 & 97 & 80,2 \\
\hline Tukvadze ${ }^{19}$ & Georgia, 2012 & 159 & 78,0 \\
\hline Este estudio & Venezuela, 2012 & 19 & 88,2 \\
\hline
\end{tabular}

$2(11,8 \%)$ de las cepas fenotípicamente resistentes a HIN no fue detectada la mutación $\mathrm{S} 315 \mathrm{~T}$ del gen $\mathrm{KatG}$.

De acuerdo a lo anterior, la detección de la mutación $\mathrm{S} 315 \mathrm{~T}$ en el gen $\mathrm{kat} G$ como estrategia para la identificación de cepas de $M$. tuberculosis resistentes a HIN exhibió una sensibilidad de $88,24 \%$, una especificidad de $100 \%$, un valor predictor positivo de $100 \%$ y un valor predictor negativo de $92 \%$.

$\mathrm{El}$ grado de concordancia entre resultados obtenidos por los métodos de las proporciones y RPC-PLRF, estimado mediante el cálculo del índice kappa, fue considerado muy bueno $($ kappa $=0,85(\mathrm{p}<0,01)$.

\section{Discusión}

La emergencia de cepas de $M$. tuberculosis resistentes a los fármacos de primera elección para su tratamiento, constituye un problema de salud en diversas partes del mundo, que amerita la implementación de programas permanentes de vigilancia. En Venezuela se dispone de un programa de control de esta enfermedad; sin embargo, los estudios de resistencia son limitados por la escasez y centralización de los recursos. Las limitadas pruebas de susceptibilidad que se realizan, basadas en métodos fenotípicos que requieren crecimiento en medios sólidos con largos períodos de incubación, son realizadas en la región capital, causando serios retrasos en el reporte de laboratorio, en la aplicación de esquemas terapéuticos efectivos y fracasos en el tratamiento, particularmente en pacientes del interior del país.

El interés en identificar oportunamente cepas resistentes a HIN se fundamenta en que generalmente la resistencia a este fármaco es la primera en surgir y suele acompañarse de resistencia a otros fármacos de primera o segunda línea (multi-resistencia), lo cual coincide con los hallazgos de este estudio, donde una gran proporción de las cepas resistentes a HIN, exhibió resistencia a RFP e indicando que este alto porcentaje de pacientes infectados con TBC-MDR debe ser tratado con antimicrobianos de segunda línea, contribuyendo a una disminución del riesgo de propagación de estas cepas, lo que obliga a aplicar medidas de control más rigurosas ${ }^{14,21,28-30}$.

En el presente estudio, que incluyó aislados clínicos de $M$. tuberculosis provenientes de siete estados del país, se obtuvo 9,8\% de resistencia a HIN. El hallazgo de un porcentaje importante de resistencia a dos o más antimicrobianos entre las cepas resistentes a HIN (70,5\%), sugiere que una gran proporción de las cepas con resistencia a este fármaco en nuestro país, corresponden a cepas TBC-MDR.

Aunque el método de las proporciones se considera el método convencional de referencia para las pruebas de 
susceptibilidad de $M$. tuberculosis, se plantea una necesidad creciente de incorporar al laboratorio de diagnóstico, nuevas estrategias que permitan acortar el período de reporte de resultado que contribuyan a contrarrestar oportunamente el problema del incremento en la transmisión de cepas resistentes a antimicrobianos.

Como respuesta a esta necesidad, se han diseñado diversas pruebas, entre ellas, una de las que parece ofrecer mayores ventajas es la técnica colorimétrica de nitrato reductasa. En el presente estudio, esta prueba exhibió una concordancia de $100 \%$ con el método de las proporciones, en lo referente a resistencia a HIN y RFP. Estos resultados son similares a los reportados por otros autores quienes refieren una excelente concordancia entre el método de nitrato reductasa directo (en muestras de esputo) y el método de las proporciones, para la detección de resistencia a HIN y RFP, los dos fármacos anti-tuberculosos más utilizados ${ }^{23,31}$.

A través del análisis genotípico de resistencia a HIN, se encontró que las cepas fenotípicamente sensibles al antimicrobiano presentaban la versión silvestre del gen $k a t G$, mientras que la mutación S315T fue detectada en $88,2 \%$ de las cepas resistentes a HIN, una frecuencia ligeramente superior a la reportada en diversos estudios (Tabla 3), demostrando la importante contribución de la mutación katG S315T a la expresión fenotípica de resistencia a HIN en los aislados clínicos de M. tuberculosis en nuestra región.

La mayoría de los reportes sobre resistencia a HIN sugieren que las mutaciones katG S315T, conjuntamente con la mutación del promotor del gen $\operatorname{inh} A$, explican la mayor parte de la resistencia a HIN, aun cuando en un pequeño porcentaje de cepas, la resistencia no puede atribuirse a alteraciones en la secuencia de estos genes ${ }^{14,15,21,33}$. Indudablemente, el estudio de susceptibilidad a HIN resulta complejo, debido a la heterogeneidad genética para el fenotipo de resistencia a este antimicrobiano; sin embargo, la mayoría de las investigaciones señalan que las mutaciones en el gen kat $G$ podría explicar aproximadamente entre 70 y $85 \%$ de la resistencia (Tabla 3), siendo de mayor importancia clínica, por inducir mayores niveles de resistencia a $\mathrm{HIN}^{25,34-36}$.

En el presente estudio, $11,8 \%$ de las cepas fenotípicamente resistentes no presentaron la mutación katG. Estas cepas presentaron resistencia múltiple, a 2 y 4 antimicrobianos, respectivamente. Aunque muy probablemente la resistencia en ellas esté asociada a mutaciones en los genes inhA y $a h p C$, es necesario realizar estudios adicionales, tales como la secuenciación de estos genes, para identificar el (los) mecanismo(s) de resistencia que estarían participando en la expresión de resistencia a HIN en estas cepas.

El hallazgo de mayor impacto de esta investigación es la demostración de que la mutación S315T parece representar un mecanismo de desarrollo de resistencia a HIN predominante en las cepas circulantes en nuestro medio; no obstante, estos datos preliminares deberán ser confirmados debido a limitaciones de este estudio en el cual no fue posible disponer de mayor número de aislados clínicos. Si estos hallazgos son confirmados, este comportamiento biológico nos brindaría la oportunidad de utilizar la detección de la mutación S315T como una herramienta diagnóstica rápida que permitiría detectar potencialmente al menos $88 \%$ de cepas resistentes, lo cual es particularmente relevante en pacientes con fracaso de tratamiento.

El método de RPC-PLFR, demostró una excelente especificidad, sensibilidad, y valores predictores positivo y negativo, al ser comparado con los resultados obtenidos por el método referencial de las proporciones.

En cuanto a la velocidad de obtención de resultados, el método genotípico supera al método fenotípico convencional, puesto que el procedimiento total (extracción de ADN, amplificación, verificación del amplicón y análisis de restricción) puede ser ejecutado en un lapso de $10 \mathrm{~h}$, a partir del cultivo primario. Otra ventaja a considerar para el método genotípico es que supera la limitación de pérdidas o retrasos por contaminación de los cultivos, así como retrasos adicionales inherentes a nuestro sistema centralizado, debido a que las pruebas de susceptibilidad son ejecutadas por los laboratorios de la región capital.

En los últimos años se han diseñado algunos ensayos altamente sensibles y específicos, basados en amplificación de ácidos nucleicos, algunos de los cuales se encuentran comercialmente disponibles (INNO-LiPA, GenXpert MTB/RIF, Genotype MTBDRplus); sin embargo, el costo de equipos y reactivos requeridos para ejecutar estas técnicas comerciales exceden la disponibilidad presupuestaria de nuestros laboratorios locales. La técnica de RPC-PLFR puede ser ejecutada utilizando una infraestructura básica de laboratorio que incluye un termociclador, tanque de electroforesis y transiluminador y suministros mínimos como partidores, $\mathrm{ADN}$ polimerasa y enzima de restricción, que pueden ser adquiridos separadamente, resultando en menor costo por ensayo.

Agradecimientos. Al laboratorio Nacional de Referencia de Tuberculosis y Enfermedades Respiratorias del Hospital El Algodonal, por el suministro de cepas de M. tuberculosis. Al Laboratorio Regional de Referencia Bacteriológica del Hospital Universitario de Maracaibo y a la Coordinación Regional del Control de la Tuberculosis del Estado Zulia por su apoyo para la realización de este trabajo.

\section{Resumen}

Introducción: La emergencia de cepas de Mycobacterium tuberculosis multi-resistentes a antimicrobianos 
constituye un problema de salud pública. Objetivo: Caracterizar la resistencia a isoniacida (HIN) en cepas de M. tuberculosis. Métodos: Se aplicaron métodos fenotípicos y genotípicos para determinar la contribución de mutaciones en el codón 315 del gen kat $G$ a la expresión fenotípica de resistencia. El análisis fenotípico de susceptibilidad a antimicrobianos se realizó por métodos de las proporciones de Canetti y nitrato reductasa. El análisis genotípico se realizó por RPC-PLFR. Resultados: Se analizaron 193 cepas de M. tuberculosis aisladas de pacientes sintomáticos respiratorios. Se identificaron 19 $(9,8 \%)$ cepas resistentes a HIN, de las cuales, $12(63,2 \%)$ exhibieron resistencia a otros antimicrobianos. El análisis genotípico permitió detectar la mutación katG $\mathrm{S} 315 \mathrm{~T}$ en 15/17 cepas fenotípicamente resistentes a HIN, exhibiendo una sensibilidad de $88,24 \%$, especificidad de $100 \%$, valor predictor positivo de $100 \%$, valor predictor negativo de $92 \%$ y alta concordancia al comparar con los métodos fenotípicos (kappa $=0,85$ ( $\mathrm{p}<0,01)$. Conclusión: La mutación S315T en el gen katG representa un mecanismo de desarrollo de resistencia a HIN predominante en las cepas circulantes en nuestro medio, característica que podría ser utilizada como herramienta diagnóstica rápida para detectar al menos $88 \%$ de las cepas resistentes a isoniacida, con gran impacto en el manejo terapéutico de los pacientes.

\section{Referencias bibliográficas}

1.- World Health Organization. Global tuberculosis control-surveillance, planning, financing. WHO Report. WHO/HTM/TB 2009: 331-9.

2.- Dye C, Scheele S, Dolin P, Pathania V, Raviglione M C. Consensus statement. Global burden of tuberculosis: estimated incidence, prevalence, and mortality by country. WHO Global Surveillance and Monitoring Project. JAMA 1999; 282: 677-86.

3.- WHO and IUATLD. The WHO/IUATLD Global project on anti-tuberculosis drug resistance surveillance 2002-2007: antituberculosis drug resistance in the world: 4 th global report. Geneva, Switzerland. WHO 2008; 223-35.

4.- Ministerio del Poder Popular para la Salud. Coordinación Nacional de Salud y Enfermedades Respiratoria. Caracas. Venezuela. Semana Epidemiológica 04-10 de marzo. Boletín Epidemiológico 2012; 10 : 4-9.

5.- Pineda M, Bonilla X, Perozo A. Boletín sobre etiología y resistencia bacteriana, Venezuela. Maracaibo. CRB.SAHUM 2009; 8: 4-511.

6.- Wang D, Yang C, Kuang T, Lei H, Meng X, Tong A, et al. Prevalence of multidrug and extensively drug-resistant tuberculosis in Beijing, China: a hospital-based retrospective study. Jpn J Infect Dis 2010; 63: 368-71.

7.- Ghebremichael S, Groenheit R, Pennhag A, Koivula T, Andersson E, Bruchfeld J, et al. Drug resistant Mycobacterium tuberculosis of the Beijing genotype does not spread in Sweden PLoS One 2010; 5:e10893.

8.- Kant S, Maurya A, Kushwaha R, Nag V, Prasad R. Multi-drug resistant tuberculosis: an iatrogenic problem. Biosci Trends 2010; 4: 48-55.

9.- Alfaresi S, Ali H. Susceptibility pattern and epidemiology of Mycobacterium tuberculosis in United Emirati hospital. Open Microbiol J 2010; 8: 1-4.
10.- Centers for Disease Control and Prevention (CDC). Revised definition of extensively drug resistant tuberculosis. MMWR Morb Mortal Wkly Rep 2006; 55: 1169-92.

11.- Riska E, Jacobs W R, Allan D. Molecular determinants of drugs resistance in tuberculosis. Int J Tuberc Lung Dis 2000; 42: S4-S10.

12.- Sharma SK, Mohan A. Multidrug-resistant tuberculosis. Indian J Med Res 2004; 120 : 354-76.

13.- Ramaswamy S, Musser J M. Molecular genetics basis of antimicrobial agent resistance in Mycobacterium tuberculosis: update. Tuberc Lung Dis 1998; 79: 3-29.

14.- Perizzolo P F, Dalla Costa E R, Ribeiro A W, Spies F S, Ribeiro M O, Dias C F, et al. Characteristics of multidrug-resistant Mycobacterium tuberculosis in Southern Brazil. Tuberculosis 2012; 92: 56-9.

15.- Arráiz N, Bermúdez V, Urdaneta B. Resistencia a drogas en M. tuberculosis: Bases moleculares. AVFT 2005; 24: 23-31.

16.- Zhang L, Ye Y, Duo L, Wang T, Song X, Lu X, et al. Application of Genotype MTBDR plus in rapid detection of the Mycobacterium tuberculosis complex as well as its resistance to isoniazid and rifampin in a high volume laboratory in Southern China. Mol Biol Rep 2011; 38: 2185-92.

17.- Ramírez M, Cowart K, Campbell $P$, Morlock G, Sikes D, Winchell J M, et al. Rapid detection of multidrug resistant tuberculosis using real-time PCR and high resolution melt analysis. J Clin Microbiol 2010; 48: 4003- 9 .

18.- Zhang Y, Young D. Transformation with $k a t G$ restores isoniazid-sensitivity in Mycobacterium tuberculosis isolates resistant to a range of drug concentrations. Mol Microbiol 1993; 8: 521-4.

19.- Tukvadze N, Kempker R, Kalandadze I, Kurbatova E, Leonard M, Apsindzelashvili $\mathrm{R}$, et al. Use of a molecular diagnostic test in AFB smear positive tuberculosis suspects greatly reduces time to detection of multidrug resistant tuberculosis. PLoS ONE 7: e31563. doi: 10.1371 .

20.- Mathuria J, Nath G, Samaria J, Anupurba S. Molecular characterization of INH-resistant Mycobacterium tuberculosis isolates by PCRRFLP and multiplex-PCR in North India. Infect Genet Evol 2009, 9: 1352-5.

21.- Telenti A, Honoré N, Bernasconi C, March J, Ortega A, Heym B, et al. Genotypic assessment of isoniazid and rifampin resistance in Mycobacterium tuberculosis: A blind study at reference laboratory level. J Clin Microbiol 1997; 35: 719-23.

22.- Murray P R, Baron E S, Pfaller M A, Tenover F C, Yolken R H. Manual of Clinical Microbiology. 9th Washington, DC. ASM Press 2007; 36: 543-8.

23.- Gupta M, Shamma N, Kaur I R. Evaluation of nitrate reductase assay for direct detection of drug resistance in Mycobacterium tuberculosis: rapid and inexpensive method for low-resource settings. Indian J Med Microbiol 2010; 28: 363-5.

24.- Mishra B, Muralidharan S, Srinivasa H. Direct drug susceptibility testing of Mycobacterium tuberculosis to primary anti-tubercular drugs by nitrate reductase assay. Indian J Pathol Microbiol 2009; 52: 343-4.

25.- Arráiz N, Romay Z, Faría N. Evaluación de un ensayo de RPC múltiple para diferenciar micobacterias del complejo Mycobacterium tuberculosis en un laboratorio de referencia. Rev Chilena Infectol 2007; 24: 99-105.

26.- Caws M, Quang D, Minh P. PCR-restriction fragment length polymorphism for rapid, low-cost identification of isoniazid-resistant Mycobacterium tuberculosis. J Clin Microbiol 2007; 45: 1789-93.

27.- Dye C, Williams B G, Espinal M A, Raviglione M C. Erasing the world's slow stain: strategies to beat multidrugresistant tuberculosis. Science 2002; 15 : 295: 2042-6.

28.- Bartu V, Kopecka E, Havelkova M. Factors associated with multidrug-resistant tuberculosis: 
comparison of patients born inside and outside of the Czech Republic J Int Med Res 2010; 38: 1156-63.

29.- Yao C, Zhu T, Zhang B, Huang J, Fu W. Detection of rpoB, kat $G$ and inhA gene mutations in Mycobacterium tuberculosis clinical isolates from Chongqing as determined by microarray. Clin Microbiol Infect 2010; 16: 1639-43.

30.- Guo H, Seet Q, Denkin S, Parsons L, Zhang Y. Molecular characterization of isoniazid-resistant clinical isolates of Mycobacterium tuberculosis from the USA. J Med Microbiol 2006; 55: 1527-31.

31.- Sethi S, Sharma S, Sharma S K, Meharwal S K, Jindal S K, Sharma M. Drug susceptibility of Mycobacterium tuberculosis to primary antitubercular drugs by nitrate reductase assay. Indian J Med Res 2004; 120: 468-71.

32.- Viader J, Luna C, Reyes J, Valdés R, del Bosque M, Tijerina M. Frequency of mutations in rpoB and codón 315 and 463 of $k a t G$ in rifampin and/or isoniazid resistant Mycobacterium tuberculosis isolates from northeast- Mejico. Microb Drug Resist 2003; 9: 33-8.

33.- Heym B, Alzari P, Honoré N, Cole S. Missense mutations in the catalase-peroxidase gene, $\mathrm{kat} G$, are associated with isoniazid resistance in Mycobacterium tuberculosis. Mol Microbiol 1995; 15: 235-45.

34.- Sangare L, Diande S, Kouanda S,
Dingtoumda B I, Mourfou A, Ouedraogo F, et al. Mycobacterium tuberculosis drugresistance in previously treated patients in Ouagadougou, Burkina Faso. Ann Afr Med 2010; 9: 15-9.

35.- Paramasivan C N, Rehman F, Wares F, Sundar Mohan N, Sundar S, et al. First- and second-line drug resistance patterns among previously treated tuberculosis patients in India. Int J Tuberc Lung Dis 2010; 14: 243-6.

36.- Ahmad S, Al-Mutairi N, Mokaddas E. Comparison of performance of two DNA line probe assays for rapid detection of multidrug-resistant isolates of Mycobacterium tuberculosis. Indian J Exp Biol 2009; 47: 45462 